University of Nebraska - Lincoln

DigitalCommons@University of Nebraska - Lincoln

Jay F. Storz Publications

Papers in the Biological Sciences

September 2002

\title{
Genetic Effective Size of a Wild Primate Population: Influence of Current and Historical Demography
}

Jay F. Storz

University of Nebraska - Lincoln, jstorz2@unl.edu

Uma Ramakrishnan

University of California at San Diego, La Jolla, California

Susan C. Alberts

Duke University, Box 90338, Durham, North Carolina

Follow this and additional works at: https://digitalcommons.unl.edu/bioscistorz

Part of the Genetics and Genomics Commons

Storz, Jay F.; Ramakrishnan, Uma; and Alberts, Susan C., "Genetic Effective Size of a Wild Primate Population: Influence of Current and Historical Demography" (2002). Jay F. Storz Publications. 17. https://digitalcommons.unl.edu/bioscistorz/17

This Article is brought to you for free and open access by the Papers in the Biological Sciences at DigitalCommons@University of Nebraska - Lincoln. It has been accepted for inclusion in Jay F. Storz Publications by an authorized administrator of DigitalCommons@University of Nebraska - Lincoln. 


\title{
Genetic Effective Size of a Wild Primate Population: Influence of Current and Historical Demography
}

\author{
Jay F. Storz, ${ }^{*}$ Uma Ramakrishnan, ${ }^{\dagger}$ and Susan C. Alberts ${ }^{\ddagger}$ \\ * Department of Ecology and Evolutionary Biology, University of Arizona, Biosciences West, Tucson, Arizona 85721, \\ ${ }^{\dagger}$ Department of Biology 0116, University of California at San Diego, La Jolla, California 92093, \\ ¥ Department of Biology, Duke University, Box 90338, Durham, North Carolina 27708
}

\begin{abstract}
A comprehensive assessment of the determinants of effective population size $\left(N_{\mathrm{e}}\right)$ requires estimates of variance in lifetime reproductive success and past changes in census numbers. For natural populations, such information can be best obtained by combining longitudinal data on individual life histories and genetic marker-based inferences of demographic history. Independent estimates of the variance effective size $\left(N_{\mathrm{eV}}\right.$, obtained from life-history data) and the inbreeding effective size $\left(N_{\mathrm{e}^{\prime}}\right.$ obtained from genetic data) provide a means of disentangling the effects of current and historical demography. The purpose of this study was to assess the demographic determinants of $N_{\mathrm{e}}$ in one of the most intensively studied natural populations of a vertebrate species: the population of savannah baboons (Papio cynocephalus) in the Amboseli Basin, southern Kenya. We tested the hypotheses that $N_{\mathrm{eV}}<N<N_{\mathrm{eI}}$ (where $N=$ population census number) due to a recent demographic bottleneck. $N_{\mathrm{eV}}$ was estimated using a stochastic demographic model based on detailed life-history data spanning a 28-year period. Using empirical estimates of age-specific rates of survival and fertility for both sexes, individual-based simulations were used to estimate the variance in lifetime reproductive success. The resultant values translated into an $N_{\mathrm{eV}} / N$ estimate of $0.329(\mathrm{SD}=0.116,95 \% \mathrm{CI}=0.172-0.537)$. Historical $N_{\mathrm{eI}}$ was estimated from 14 -locus microsatellite genotypes using a coalescent-based simulation model. Estimates of $N_{\mathrm{el}}$ were 2.2 to 7.2 times higher than the contemporary census number of the Amboseli baboon population. In addition to the effects of immigration, the disparity between historical $N_{\text {el }}$ and contemporary $N$ is likely attributable to the time lag between the recent drop in census numbers and the rate of increase in the average probability of allelic identity-by-descent. Thus, observed levels of genetic diversity may primarily reflect the population's prebottleneck history rather than its current demography.
\end{abstract}

Keywords: Coalescent, conservation genetics, effective population size, genetic bottleneck, individual-based model, microsatellite DNA, Papio cynocephalus, reproductive success

Genetically effective population size $\left(N_{\mathrm{e}}\right)$ is determined by the net effect of individual life histories (i.e., variance in lifetime reproductive success) and demographic history (i.e., temporal fluctuations in census numbers; Wright 1938; Caballero 1994). For natural populations of vertebrate species, reasonably comprehensive information about lifetime reproductive success can only be obtained from intensive longitudinal studies of individually known animals. Such studies therefore provide the best opportunities for assessing the demographic determinants of $N_{\mathrm{e}}$ and the relationship between $N_{\mathrm{e}}$ and population census number $(N)$. Estimating $N_{\mathrm{e}}$ of natural populations is critically important for making inferences about the past and present course of microevolutionary change (Lande and Barrowclough 1987). The standing level of genetic variation determines a population's capacity for adaptive response to novel selection pressures and can also strongly influence extinction risk of remnant populations (Mills and Smouse 1994; Lande 1995; Lynch et al. 1995; Whitlock 2000; Higgins and Lynch 2001). Thus, $N_{\mathrm{e}}$ also serves as an important indicator of population viability (Lande and Barrowclough 1987; Nunney and Campbell 1993).

Effective population size is defined as the size of an idealized population that would give rise to the same temporal variance in allelic frequencies (variance effective size, $N_{\mathrm{eV}}$ ) or the same rate of increase in the probability of allelic identity-by-descent (inbreeding effective size, $N_{\mathrm{eI}}$ ) as in the actual population under consideration (Wright 1931, 1938; Kimura and Crow 1963; Crow and Denniston 1988; Cabal- lero 1994). In a randomly mating population of constant size, $N_{\mathrm{eV}}$ and $N_{\mathrm{el}}$ are expected to be identical (Hill 1972; Ewens 1982; Caballero 1994). However, a recent demographic expansion or contraction can produce a substantial discrepancy between the two measures because $N_{\mathrm{eV}}$ is related to the number of descendents, whereas $N_{\mathrm{eI}}$ is related to the number of ancestors (Templeton 1980; Crow and Denniston 1988; Templeton and Read 1994).

Effective sizes of natural populations have been estimated using both genetic and demographic approaches, but only rarely in combination (Begon et al. 1980; Husband and Barrett 1992; Bouteiller and Perrin 2000). DNA polymorphism data provide a means of estimating historical $N_{\mathrm{eI}}$ using theory that relates coalescence times of allelic lineages to long-term rates of drift (Felsenstein 1992a,b; Fu 1994a,b; Kuhner et al. 1995, 1998; Nielsen 1997). Genetic methods offer a retrospective view of the rate of drift over past generations, but provide no insight into the causal factors underlying any particular value of $N_{\mathrm{el}}$. In contrast, demographic methods can be used to estimate $N_{\mathrm{eV}}$ of contemporary populations by measuring parameters that influence variance in lifetime reproductive success. Such methods provide a view of the prospective rate of drift and can be used to predict the direction and magnitude of changes in $N_{\mathrm{eV}}$ that result from variation in ecological conditions (Nunney and Elam 1994). Comparing estimates of $N_{\mathrm{eI}}$ and $N_{\mathrm{eV}}$ can thus be used to make inferences about the effects of recent demographic changes on levels of genetic diversity (Crandall et al. 1999). A recent demographic contraction is expected 
to reduce $N_{\mathrm{eI}}$ relative to $N_{\mathrm{eV}}$. In contrast, a recent demographic expansion can reduce $N_{\mathrm{eV}}$ relative to $N_{\mathrm{eI}}$ (Templeton 1980; Crow and Denniston 1988; Templeton and Read 1994; Gerber and Templeton 1996).

The purpose of this study was to assess the demographic determinants of $N_{\mathrm{e}}$ in one of the most intensively studied natural populations of a vertebrate species: the population of savannah baboons (Papio cynocephalus) in the Amboseli Basin, southern Kenya. Specifically, we tested the hypothesis that $N_{\mathrm{eV}}<N<N_{\mathrm{el}}$ due to a recent demographic contraction (Altmann et al. 1985; Samuels and Altmann 1991). $N_{\mathrm{eV}}$ was estimated using a stochastic demographic model based on detailed life-history data spanning a 28-year period. $N_{\text {eI }}$ was estimated from 14-locus microsatellite genotypes using a coalescent-based simulation model. Independent estimates of $N_{\mathrm{eV}}$ and $N_{\mathrm{el}}$ were used to disentangle the effects of current and historical demographic processes.

\section{Materials and Methods}

\section{Study Population}

The Amboseli Basin is an area of semiarid savannah in southern Kenya (for details see Western and van Praet 1973; Western 1983). Census data indicate that a stable population of approximately 480 baboons currently resides within the Amboseli Basin (Samuels and Altmann 1991). This represents about an $81 \%$ reduction relative to the estimated census number in 1963-1964 ( 2500 baboons; Altmann et al. 1985). The Amboseli baboon population underwent a dramatic demographic bottleneck in the late 1960s and early 1970s, coinciding with drought-induced habitat changes (Altmann et al. 1985). In the space of several years, Amboseli was transformed from an Acacia woodland/ grassland mosaic to a more xeric savannah community (Western and van Praet 1973). In addition to a reduction in the overall quality of forage, the Amboseli baboon population suffered from massive die-offs of fever trees (Acacia xanthophloea), which provide important sleeping sites and refuge from predators (Altmann et al. 1985). Census numbers of Amboseli baboons stabilized by the mid-1970s (Samuels and Altmann 1991; Alberts and Altmann 2002).

Dispersal to and from the basin is restricted by ecological and physiographic barriers. To the north and west, the Amboseli baboon population is separated from other populations by $45-80 \mathrm{~km}$ of inhospitable terrain. The range of hills to the east represents the most common route of immigration into the Amboseli Basin (Samuels and Altmann 1991). All such immigration is exclusively male mediated (Alberts and Altmann 1995).

\section{Demographic Analysis}

$N_{\mathrm{eV}}$ of the Amboseli baboon population was estimated using a stochastic demographic model. Using empirical estimates of vital rates (age-specific rates of survival and reproduction), individual-based simulations were used to estimate the variance in lifetime reproductive success. The resultant values were then used to estimate the ratio $N_{\mathrm{eV}} / N$.

\section{Estimation of vital rates.}

Age-specific rates of survival and fertility were estimated separately for males and females due to sex differences in age distribution and reproductive patterns. For both sexes, estimates of annual rates of survival and fertility were extracted from continuous observational data collected from 1971 to 1999. Age-classes represented 1-year time intervals, resulting in 27 age classes for females and 21 for males. Because many individuals in the population were still alive at the time of the final census (December 31, 1999), the analysis included incomplete life histories (censored data). Inclusion of censored data is important because the sample of complete life histories is potentially biased toward short life spans (Alberts and Altmann 2002).

For each sex, age-specific survivorship was estimated using censored data by employing Kaplan-Meier estimates of survival in each age class (Kalbfleisch and Prentice 1980; Lee 1992; Allison 1995). With this method, age-specific hazard rates (Allison 1995; Alberts and Altmann 2002) were calculated as

$$
h_{i}=\left(N_{i} / T_{i}\right) \text {, }
$$

where $N_{i}$ is the number of individuals that died in the $i$ th age-class and $T_{i}$ is the cumulative length of time that all individuals spent in the $i$ th age-class $(<1$ year for individuals that died or were censored in age class $i$ ). Survivorship (the probability of surviving from birth to the $i$ th birthday) was then calculated as

$$
l(i)=\left[1-\left(N_{i} / T_{i}\right)\right][1(i-1)] .
$$

Because baboons are continuous, nonseasonal breeders, we used birth-flow estimates of age-specific survival rates (proportion of individuals that survive age class $i$, conditional on having entered age class $i$ ) as described in Caswell (2001):

$$
P_{i}=[l(i)+l(i+1)] \div[l(i-1)+l(i)] .
$$

For males, $P_{i}$ of postdispersal age classes (seven and higher) was estimated from the population age distribution (see Alberts and Altmann 2002). Estimates of age- and sex-specific rates of survival were based on data for 277 deaths that occurred during 2438.10 baboon-years of exposure between 1971 and 1999.

Birth rate was calculated as $m_{i}=B_{i} / N_{i}$, where $B_{i}$ is the number of births to females (or newborns sired by males) and $N_{i}$ is the number of individuals who either survived through age class $i$ or who successfully reproduced within age class $i$ but died (or were censored) before making the transition to age class $i+1$. Following Caswell (2001), fertility of a particular age class (expected number of offspring produced by an individual entering age class $i$ ) was estimated as

$$
F_{i}=l(0.5)\left(m_{i}+P_{i} m_{i+1}\right) / 2 .
$$

Inclusion of the term $l(0.5)$ accounts for the fact that, on average, a newborn will have to survive through half the census interval in its year of birth to be included in the next census. For males, reproductive data were obtained from re- 
cords of mate guarding (consortships). The use of consortships as a proxy for male mating success has been validated by genetic paternity data (Altmann et al. 1996). Birth rates were calculated as the number of male newborns that were sired by males in each age class divided by the total number of male years per age class. Estimates of age- and sexspecific rates of fertility were based on 267 female offspring born to females and 233 male offspring sired by males.

\section{Estimation of lifetime reproductive success and $N_{e V} / N$.}

Calculation of $N_{\mathrm{eV}} / N$ requires estimation of the variance in lifetime reproductive success for both sexes over the course of a complete life cycle (e.g., newborn to newborn). To estimate $N_{\mathrm{eV}} / N$ of the Amboseli baboon population, we used an individual-based simulation model to estimate the variance in lifetime reproductive success for a population of 480 animals (= contemporary census number; Samuels and Altmann 1991). The number of newborns produced by each member of a cohort of newborns was simulated using empirical probability distributions of age-specific fertility and survival for both sexes.

Simulations were initiated under conditions of demographic equilibrium. Survivorship curves for both sexes were used to estimate the stable age distribution, which was then used as a basis for sex and age assignments in the simulations. All newborns in the initial cohort were recorded as members of the same generation, and numbers of male and female newborns were recorded as census numbers for each sex. Survival and reproduction were assessed for each individual over a period of 27 years, the maximum observed life span of a female baboon in Amboseli. The probability of reproduction by a given female in each year of that female's life span was set equal to the age-specific fertility rate. In a similar manner, apportionment of the resultant offspring among males was determined by the normalized probability distribution of age-specific male fertility. Individual identities of newborns were recorded as part of the individual profiles of their parents. For both males and females, the duration of each individual's life span was determined by the age- and sex-specific probability distributions of survival. Deaths were recorded to assess lifetime reproductive success at the end of each simulation. Annual progeny production was summed over the total number of years comprising each individual's reproductive life span to obtain the net lifetime production of male and female progeny by parents of each sex.

Using the Hill $(1972,1979)$ equation, $N_{\mathrm{eV}}$ of the Amboseli baboon population was estimated as

$$
\begin{aligned}
& \frac{1}{N_{\mathrm{eV}}}=\frac{1}{16 N_{m}}[2+\operatorname{var}\left(X_{m m}\right)+2\left(\frac{N_{m}}{N_{f}}\right) \operatorname{cov}\left(X_{m m, m f}\right) \\
&\left.+\left(\frac{N_{m}}{N_{f}}\right)^{2} \operatorname{var}\left(X_{m f}\right)\right] \\
&+\frac{1}{16 N_{f}}\left[2+\operatorname{var}\left(X_{f f}\right)+2\left(\frac{N_{f}}{N_{m}}\right) \operatorname{cov}\left(X_{f m, f f}\right)\right. \\
&\left.+\left(\frac{N_{f}}{N_{m}}\right)^{2} \operatorname{var}\left(X_{f m}\right)\right]
\end{aligned}
$$

where $N_{i}$ is the number of $i$-sexed individuals entering the population each generation, and $X_{i j}$ is the net lifetime production of $j$-sexed progeny by $i$-sexed parents (with $m$ and $f$ denoting males and females, respectively). The Hill (1972, 1979) equation was designed specifically for age-structured populations under conditions of demographic equilibrium (see also Felsenstein 1971; Johnson 1977; Emigh and Pollak 1979; Pollak 1980). Simulations were repeated 2000 times to investigate the effects of demographic stochasticity on $N_{\mathrm{eV}}$.

\section{Sensitivity analysis.}

Because the Hill $(1972,1979)$ equation is based on the assumption of stable age structure, we tested the sensitivity of $N_{\mathrm{eV}} / N$ to departures from a stable age structure that may have been caused by the recent demographic bottleneck. Starting with a population of $N=2500$ under conditions of demographic equilibrium, the bottleneck was simulated as a single-step reduction in population size by sampling 480 individuals without replacement. We considered two distinct mortality regimes in separate simulations. In the first scenario, mortality during the bottleneck was independent of age and sex. In the second scenario, mortality during the bottleneck was two times higher for juveniles than for breeding adults (see Altmann et al. 1985), but was otherwise independent of age and sex.

\section{Genetic Analysis}

\section{Genotyping.}

A total of 136 baboons were shot with tranquilizer darts for the purpose of collecting blood samples (Altmann et al. 1996). Genomic DNA was isolated from blood samples according to the protocol described by Bruford et al. (1992) or by using QIAamp extraction columns (Qiagen, Valencia, CA). The genetic analysis was based on a total of 14 autosomal microsatellites (10 di- and four tetranucleotide repeat loci) and two allozymes (transferrin and albumin). Baboon microsatellites were amplified using primers designed from flanking sequence of homologous loci in the human genome (map positions D7S503, D13S159, D6S311, D6S271, D16S402, D4S431, D2S141, D16S420, D11S925, D17S791, D5S1457, D10S611, D14S306, and D4S243). Protocols used to assay variation at dinucleotide microsatellites and allozymes were reported previously (Altmann et al. 1996). For the tetranucleotide repeat loci, polymerase chain reaction (PCR) was performed using $8 \mu \mathrm{M}$ of each primer, $8 \mathrm{mM}$ dNTPs, $25 \mathrm{mM} \mathrm{MgCl}, 0.025$ units of AmpliTaq DNA polymerase (Perkin Elmer, Foster City, CA), 10;ts PCR buffer (100 mM Tris-HCl buffer, $\mathrm{pH} 8.3,500 \mathrm{mM} \mathrm{KCl}), \mathrm{ddH}_{2} \mathrm{O}$, and $\sim 10 \mathrm{ng}$ of template DNA in a total reaction volume of $25 \mu \mathrm{l}$. PCR was performed in an PTC-200 thermal cycler (MJ Research, Watertown, MA) under the following conditions: initial denaturation at $94^{\circ} \mathrm{C}$ for 2 min followed by 35 cycles of denaturation at $94^{\circ} \mathrm{C}$ for $45 \mathrm{sec}$, annealing at $57^{\circ} \mathrm{C}$ for $1 \mathrm{~min}$, and extension at $72^{\circ} \mathrm{C}$ for $90 \mathrm{sec}$ (with a final extension at $72^{\circ} \mathrm{C}$ for $10 \mathrm{~min}$ ). Genotyping results for dinucleotide repeat microsatellite loci and allozymes were reported in a previous analysis of 76 individuals that were also included in the present study (Altmann et al. 1996). All 
sampled individuals were typed at four tetranucleotide repeat loci, and a subset of previously unsampled individuals (not included in the study of Altmann et al. 1996) was also typed at the 10 dinucleotide repeat loci. Allele sizes were quantified using an ABI 3700 automated sequencer and analyzed using GENESCAN software (PE Biosystems, Foster City, CA). Allele sizes for dinucleotide repeat loci were standardized across datasets by retyping a sample of 25 individuals that had been previously genotyped using autoradiographic visualization of $\left[\gamma^{-}{ }^{32} \mathrm{P}\right]$-labeled PCR products in denaturing acrylamide gels (Altmann et al. 1996). Results compatible with the ABI 3700 scores were obtained by subtracting a constant number from the autoradiographic scores and rounding to the nearest integer.

\section{Tests of allelic and genotypic disequilibria.}

Linkage disequilibrium was tested for each pairwise combination of loci using an exact probability test for $\mathrm{R} \times$ C contingency tables (Raymond and Rousset 1995a). Unbiased estimates of exact probabilities were obtained using the Markov chain algorithm of Guo and Thompson (1992), as implemented in the program GENEPOP 3.1d (updated from Raymond and Rousset 1995b). The null hypothesis of Hardy-Weinberg genotypic proportions was tested by means of a randomization procedure. A null distribution of Cockerham's (1969) inbreeding coefficient $f$ was generated from 10,000 randomizations of alleles among individuals. Two-tailed probability tests were used to assess statistical significance of observed values relative to null distributions because different forms of nonrandom mating are expected to cause deviations from Hardy-Weinberg proportions in opposite directions (a heterozygote deficit may be produced by inbreeding and/or a Wahlund effect due to population subdivision, whereas a heterozygote excess may be produced by intrasexual variance in reproductive success and/or sex differences in allelic frequencies; Storz et al. 2001a). The program FSTAT 2.9.1 (updated from Goudet 1995) was used for all calculations. Expected frequencies of null alleles at each locus were estimated according to the method of Summers and Amos (1996).

\section{Tests for evidence of a recent genetic bottleneck.}

Static estimates of $N_{\mathrm{eV}}$ can be considered indicative of long-term rates of drift provided that population census numbers remain stable through time. Because temporal fluctuations in population size can result in a substantial reduction in $N_{\mathrm{eV}}$ (Wright 1938; Motro and Thomson 1982; Vucetich et al. 1997), it is important to know whether the Amboseli baboon population has experienced a recent genetic bottleneck. The occurrence of a bottleneck in the relatively recent past is also expected to produce departures from mutation-drift equilibrium, thereby invalidating an important assumption of genetic methods for estimating $N_{\mathrm{eI}}$. To test for evidence of a recent genetic bottleneck in the Amboseli baboon population, we used three different methods based on microsatellite data: two nonparametric tests of heterozygosity excess (Cornuet and Luikart 1996; Luikart and Cornuet 1998) and one graphical test based on the distribution of allelic frequencies (Luikart et al. 1998).
The first two tests are based on theory developed for neutral loci that relates the expected heterozygosity at mutation-drift equilibrium to the observed number of alleles in a population sample (Ewens 1972; Nei et al. 1975; Watterson 1984; Maruyama and Fuerst 1985; Cornuet and Luikart 1996). Population bottlenecks typically entail a rapid increase in the rate of drift, which is reflected in a reduction in $N_{\mathrm{eV}}$. Because allelic diversity is reduced more rapidly than heterozygosity during a bottleneck (Nei et al. 1975; Leberg 1992), the resultant departure from mutationdrift equilibrium produces a transient excess of HardyWeinberg heterozygosity $\left(H_{\mathrm{e}}\right)$ relative to the heterozygosity computed from the number of alleles in a population sample ( $H_{\mathrm{eq}}$; Watterson 1984; Maruyama and Fuerst 1985). It is important to note the distinction between a test for excess levels of heterozygosity $\left(H_{\mathrm{e}}>H_{\mathrm{eq}}\right)$ and a test for excess numbers of heterozygotes $\left(H_{\mathrm{o}}>H_{\mathrm{e}}\right)$. The former test compares the observed heterozygosity (or gene diversity; Nei 1987) with that expected at mutation-drift equilibrium, whereas the latter test compares the observed number of heterozygotes with that expected at Hardy-Weinberg equilibrium. The characteristic pattern of heterozygosity excess produced by a bottleneck may persist at a detectable level for $2 N_{\mathrm{eV}}$ to $4 N_{\mathrm{eV}}$ generations (where $N_{\mathrm{eV}}$ is the variance effective size of the bottlenecked population; Cornuet and Luikart 1996). The time required to attain a new mutationdrift equilibrium at the postbottleneck population size will depend on the rate and mode of mutation at the loci under consideration, as well as the severity and duration of the bottleneck (Nei et al. 1975; Cornuet and Luikart 1996).

The conclusion that bottlenecks cause a heterozygosity excess was originally based on theory developed for the infinite alleles model of mutation (Watterson 1984, 1986; Maruyama and Fuerst 1985). Cornuet and Luikart (1996) demonstrated that the conclusion can be generalized to other mutation models appropriate for microsatellite loci. Available evidence suggests that mutational dynamics of microsatellites may be best characterized by a modified form of the stepwise mutation model (SMM; Di Rienzo et al. 1994; Estoup and Cornuet 1999; Ellegren 2000). Under the strict SMM, microsatellite mutations change the state of an allele by +1 or -1 repeat unit with equal probability (Shriver et al. 1993; Valdes et al. 1993). Under the two-phase model (TPM) of Di Rienzo et al. (1994), the SMM is generalized to include a specified proportion of multistep mutations. Although Chakraborty and Nei (1977) presented a model describing bottleneck-induced changes in heterozygosity for loci under the SMM, there are no formulas available for calculating the expected number of alleles in a sample. We therefore used the coalescent-based simulation approach of Cornuet and Luikart (1996) to calculate $H_{\mathrm{eq}}$ under two separate parameterizations of the TPM. In separate simulations, the proportion of multistep mutations $(q)$ was set at 0.05 and 0.10 . In each case the size distribution of multistep mutations was characterized by a variance of 15 .

Coalescent simulations were used to generate a distribution of heterozygosity $\left(H_{\mathrm{eq}}\right)$ expected from the observed number of alleles $\left(k_{\mathrm{o}}\right)$, given the sample size $(n)$ under the assumption of mutation-drift equilibrium. Using the approach described by Cornuet and Luikart (1996), the co- 
alescent process was simulated by sampling from a likelihood distribution of $\theta$-values. The distribution of expected heterozygosity at mutation-drift equilibrium was derived from the subset of iterations that produced exactly $k_{\mathrm{o}}$ alleles. Average $H_{\mathrm{eq}}$-values were then computed for each locus and compared to the heterozygosity $\left(H_{\mathrm{e}}\right)$.

Under neutrality in a population at mutation-drift equilibrium, about $50 \%$ of sampled loci are expected to exhibit a slight excess of heterozygosity $\left(H_{\mathrm{e}}>H_{\mathrm{eq}}\right)$ and about $50 \%$ should exhibit a slight deficit of heterozygosity $\left(H_{\mathrm{e}}<H_{\mathrm{eq}}\right)$. Thus, a recent bottleneck is indicated if a disproportionate number of loci exhibit a heterozygosity excess. To determine whether the Amboseli baboon population was characterized by a significant number of loci with heterozygosity excess, we employed two statistical tests: a sign test and a Wilcoxon signed-ranks test (Cornuet and Luikart 1996; Luikart and Cornuet 1998). Because a recent population expansion is also expected to produce a departure from mutation-drift equilibrium (in the direction of a heterozygosity deficit), two-tailed probability values were used to assess statistical significance of the Wilcoxon test.

The third test used to detect a recent genetic bottleneck is based on the expected distribution of allelic frequencies at mutation-drift equilibrium. Under neutrality, alleles at low frequency $(<0.01)$ are expected to far outnumber those at intermediate frequencies, regardless of the underlying mutation model (Nei et al. 1976; Chakraborty et al. 1980; Luikart et al. 1998). Thus, a recent genetic bottleneck can be detected by a characteristic shift in modal allele counts from low to intermediate frequency classes (Luikart et al. 1998). The three tests of heterozygosity excess were based exclusively on microsatellite data. All three tests were implemented by the program BOTTLENECK (ver. 1.2.02, Piry et al. 1999).

The above tests assume that all sampled individuals represent direct descendents of the bottlenecked population. The power of the tests to detect a genetic bottleneck could be compromised if the postbottleneck sample of genotypes included a small number of newly arrived immigrants derived from a genetically distinct source population. The introduction of rare alleles by such immigrants could mask the genetic signature of a bottleneck (Keller et al. 2001). Fortunately, long-term longitudinal data allow us to assess this possibility. With the exception of 12 males, all sampled individuals were born on known dates to mothers with known matrilineal ancestry. The 12 males of unknown origin probably immigrated into the study population from elsewhere in the Amboseli Basin, but we cannot rule out the possibility that they had emigrated from more distant populations. The same tests described above were repeated after excluding these 12 males.

\section{Estimation of $\mathrm{N}_{\mathrm{el}}$.}

For each microsatellite locus, a maximum-likelihood estimate of $\theta$ was obtained using the Markov chain Monte Carlo simulation approach of Nielsen (1997; see also Griffiths and Tavaré 1994a,b,c). Under the TPM, allelic genealogies were simulated under the assumption of a neutral coalescent model with Wright-Fisher sampling between generations (Tavaré 1984; Hudson 1990). Estimates of the joint likelihood surface of $\theta$ and $q$ were based on
Table 1. Sex- and age-specific rates of survival and fertility in the Amboseli population of savannah baboons (Papio cynocephalus).

\begin{tabular}{|c|c|c|c|c|}
\hline \multirow{2}{*}{$\begin{array}{l}\text { Age } \\
\text { Class }\end{array}$} & \multicolumn{2}{|c|}{ Females } & \multicolumn{2}{|c|}{ Males } \\
\hline & Survival & Fertility & Survival & Fertility \\
\hline 1 & 0.7938 & 0.0000 & 0.7825 & 0.0000 \\
\hline 2 & 0.8884 & 0.0000 & 0.9122 & 0.0000 \\
\hline 3 & 0.9366 & 0.0000 & 0.9337 & 0.0000 \\
\hline 4 & 0.9688 & 0.0000 & 0.9167 & 0.0000 \\
\hline 5 & 0.9529 & 0.1284 & 0.9588 & 0.0000 \\
\hline 6 & 0.9439 & 0.2727 & 0.9427 & 0.0000 \\
\hline 7 & 0.9481 & 0.2755 & 0.9390 & 0.0267 \\
\hline 8 & 0.9483 & 0.2647 & 0.9416 & 0.2593 \\
\hline 9 & 0.9427 & 0.2598 & 0.9311 & 0.5688 \\
\hline 10 & 0.9233 & 0.2237 & 0.9112 & 0.5996 \\
\hline 11 & 0.8910 & 0.1981 & 0.8944 & 0.5883 \\
\hline 12 & 0.8943 & 0.2310 & 0.8820 & 0.5567 \\
\hline 13 & 0.9419 & 0.2615 & 0.8456 & 0.4134 \\
\hline 14 & 0.9160 & 0.2596 & 0.8295 & 0.3159 \\
\hline 15 & 0.8701 & 0.2842 & 0.7816 & 0.2389 \\
\hline 16 & 0.8731 & 0.3010 & 0.6665 & 0.1638 \\
\hline 17 & 0.7880 & 0.2656 & 0.6251 & 0.0503 \\
\hline 18 & 0.6329 & 0.2169 & 0.8002 & 0.0441 \\
\hline 19 & 0.6278 & 0.2440 & 0.7500 & 0.0372 \\
\hline 20 & 0.8648 & 0.1838 & 0.3333 & 0.0331 \\
\hline 21 & 0.7455 & 0.2398 & 0.0000 & 0.0000 \\
\hline 22 & 0.5517 & 0.3216 & & \\
\hline 23 & 0.6090 & 0.1306 & & \\
\hline 24 & 0.4174 & 0.2144 & & \\
\hline 25 & 0.3796 & 0.0000 & & \\
\hline 26 & 0.5000 & 0.0000 & & \\
\hline 27 & 0.0000 & 0.0000 & & \\
\hline
\end{tabular}

100,000 runs through the Markov chain. The range of possible $q$ values was restricted to $0.01-0.50$. For each locus, a moment estimate of $\theta$ (Wehrhahn 1975) was used to drive the simulations. Using maximum-likelihood estimates of $\theta$ for loci with $H_{\mathrm{e}}=0.50-0.90$ (i.e., all loci except D4S431 and D2S141) and assuming $\mu$ in the range $2 \times 10^{-3}$ to $6 \times 10^{-4}$ (Brinkman et al. 1998; Ellegren 2000), historical $N_{\text {eI }}$ was calculated as $\theta(4 \mu)^{-1}$.

\section{Results}

\section{Demographic Analysis}

Variance in lifetime reproductive success and $N_{e V} / N$.

Estimated vital rates for the Amboseli baboon population revealed a substantial degree of age-specific variation in both survival and fertility (Table 1). A total of 2000 Monte Carlo simulation trials were used to generate distributions of sex-specific estimates of variance in lifetime reproductive success (Figure 1). Simulation-based estimates of variance in lifetime reproductive success averaged 11.410 for males (SD $=5.202,95 \% \mathrm{CI}=4.110-20.664)$ and 8.445 for females $(\mathrm{SD}=3.401,95 \% \mathrm{CI}=2.993-14.339)$. This disparity between the sexes was primarily due to the high age-dependence of male reproductive success (Table 1). The resultant estimates of $N_{\mathrm{eV}} / N$ averaged $0.329(\mathrm{SD}=0.116,95 \% \mathrm{CI}$ $=0.172-0.537$; Figure 2). Results of the sensitivity analysis indicated that a bottleneck-induced departure from a stable age distribution had no significant effect on $N_{\mathrm{eV}} / \mathrm{N}$ under either of the simulated mortality schemes. Under conditions of age- and sex-independent mortality, $N_{\mathrm{eV}} / N$ of the 


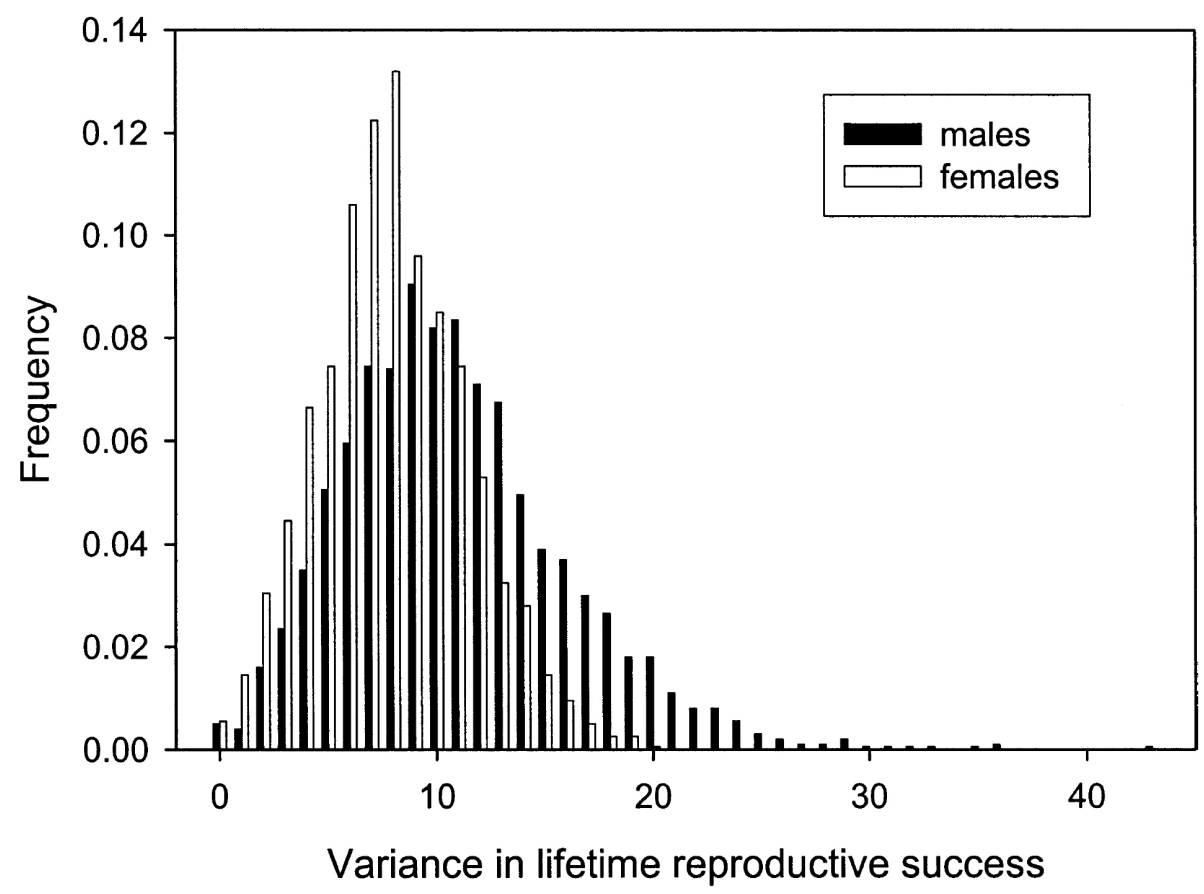

Figure 1. Distribution of simulation-based estimates of the variance in lifetime reproductive success for male and female savannah baboons (P. cynocephalus). Distributions were generated from 2000 Monte Carlo trials using an individual-based model (see text for details).

postbottleneck population averaged $0.332(\mathrm{SD}=0.119,95 \%$ $\mathrm{CI}=0.178-0.550)$. Under conditions where mortality of prereproductive age classes was twice that of adults, $N_{\mathrm{eV}} / N$ averaged $0.285(\mathrm{SD}=0.116,95 \% \mathrm{CI}=0.142-0.499)$.

\section{Genetic Analysis}

For the total sample of $P$. cynocephalus $(n=136)$, number of alleles per locus averaged 7.7 (range $=3-11$ ) and $H_{\mathrm{e}}$ averaged 0.678 (range $=0.271-0.830$; Table 2 ). Allelic frequencies for all 14 microsatellite loci are given in Table 3. Using a Bonferroni-corrected a-level of 0.0004, a statistically significant degree of two-locus linkage disequilibrium was

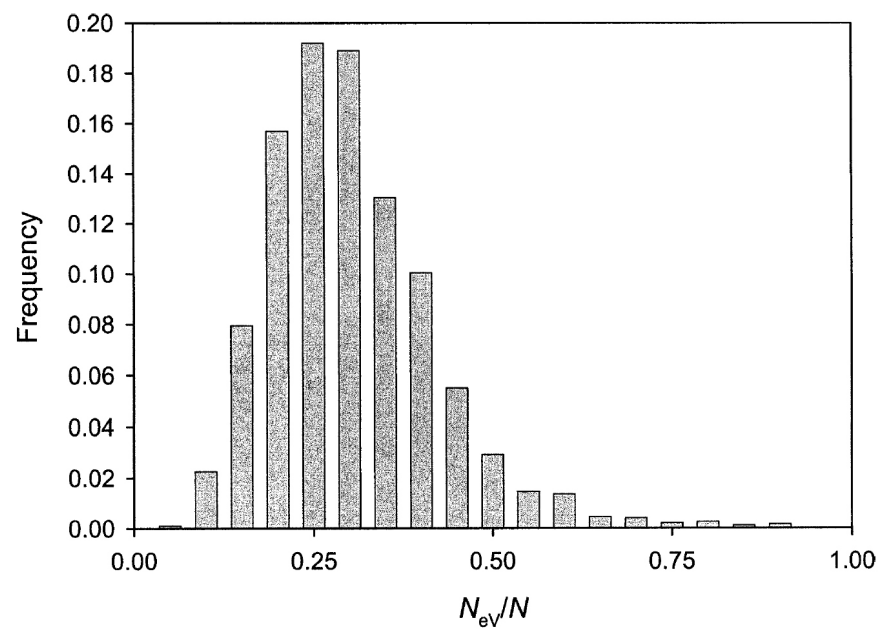

Figure 2. Distribution of simulation-based estimates of $N_{\mathrm{eV}} /$ $N$ for the Amboseli population of savannah baboons, based on 2000 Monte Carlo trials. indicated for three of 120 pairwise comparisons (D7S503 and D6S311, $P<0.0001$; D13S159 and D6S311, $P<0.0001$; and D13S159 and D6S271, $P<0.0001)$. None of these three pairwise comparisons involved linked loci (Table 2). To assess the extent to which nonindependence among loci affected parameter estimation, all multilocus analyses were repeated after eliminating D6S311 and D13S59 from the dataset. No significant departures from Hardy-Weinberg genotypic proportions were observed at any loci. The overall inbreeding coefficient $f$ (estimated from the combined set of microsatellite and allozyme loci) was -0.016 . The proportion of randomizations that yielded a smaller (more negative) $f$ than the observed value was 0.131 . Tests based on allelic and genotypic frequencies indicated that null alleles were either nonexistent or present at negligible frequencies (Table 2).

\section{Tests for evidence of a recent genetic bottleneck.}

Under the assumption that all loci could be adequately characterized by the TPM (with $q=0.05-0.10$ ), eight of 14 loci exhibited a heterozygosity deficit and six of 14 exhibited a heterozygosity excess relative to the average expected value at mutation-drift equilibrium (Table 4; Figure $3)$. The sign test revealed that these proportions were not significantly different from the null expectation of a 1:1 ratio $(P=0.166$ and 0.161 for $q=0.05$ and 0.10 , respectively). Likewise, the result of the Wilcoxon signed rank test was not statistically significant for $q=0.10(P=0.135)$ but approached significance for $q=0.05(P=0.068)$. The graphical method of Luikart et al. (1998) revealed no mode-shift distortion in the distribution of allelic frequencies (Figure 4). The relative number of rare alleles at low frequency was consistent with the expected distribution for neutral loci at mutation-drift equilibrium (Luikart et al. 1998). The null 
Table 2. Summary statistics for microsatellite variation in the Amboseli population of savannah baboons, Papio cynocephalus. Assignments of microsatellite loci to baboon chromosomes (following karyotypic nomenclature of Cambefort et al. 1976) were based on linkage map data from Rogers et al. (2000). $n$, number of chromosomes sampled per locus, $k_{\mathrm{o}^{\prime}}$ number of alleles per locus; $H_{\mathrm{o}^{\prime}}$ observed heterozygosity, $H_{\mathrm{e}^{\prime}}$ expected heterozygosity at Hardy-Weinberg equilbrium; $f$, Cockerham's (1969) inbreeding coefficient; and $f_{0}$, expected frequency of null alleles.

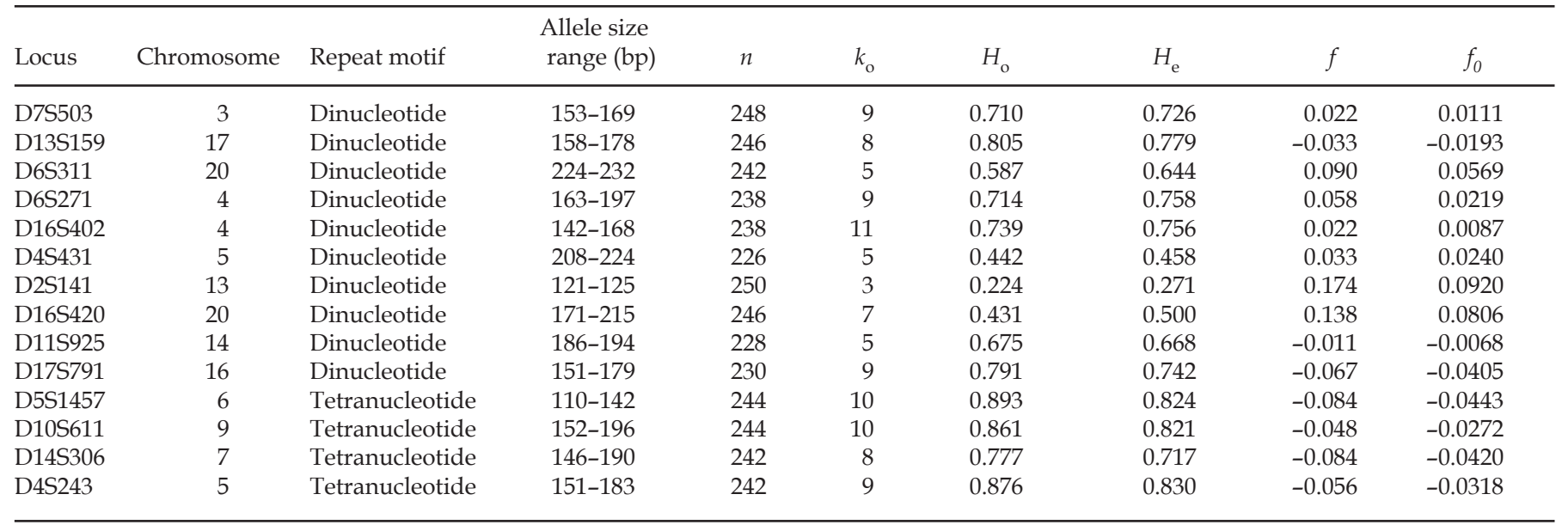

hypothesis of mutation-drift equilibrium was not rejected by any of the three tests. Results were not affected by eliminating D6S311 and D13S59 (loci involved in three cases of pairwise linkage disequilibrium), nor were they affected by eliminating immigrant males from the dataset.

\section{Estimation of $N_{e I^{*}}$.}

For the 12 loci with $H_{\mathrm{e}}=0.50-0.90$, maximum-likelihood estimates of $\theta$ under the TPM averaged 8.293 (range = 2.377-18.360; Table 5). This average value translates into $N_{\mathrm{eI}}$ in the range 1037-3456, assuming $\mu$ in the range $2 \times 10^{-3}$ to $6 \times 10^{-4}$. Maximum-likelihood estimates of $q$ (proportion of multistep mutations) averaged 0.068 for the same 12 loci (range $=0.010-0.476$; Table 5) .

\section{Discussion}

Results of the stochastic demographic model indicated that $N_{\mathrm{eV}}$ of the Amboseli baboon population was approximately one-third of the total census number. In contrast, the coalescent-based analysis of microsatellite variation indicated that long-term $N_{\mathrm{eI}}$ was 2.2 to 7.2 times higher than the contemporary census number within the Amboseli Basin. Based on available information about the recent demographic history of the Amboseli baboon population, these results are consistent with the expected rank order of effective sizes relative to census number $\left(N_{\mathrm{eV}}<N<N_{\mathrm{eI}}\right)$.

\section{Current Demography and $N_{e V} / N$}

Papio cynocephalus is one of the most polygynous and most sexually dimorphic primate species, suggesting that variance in male reproductive success could result in a substantial reduction of $N_{\mathrm{eV}}$. However, the effective reproductive life spans of males are typically compressed into a small portion of their total adult life span (Altmann et al. 1996; Alberts and Altmann 2002; see Table 1). Thus, high variance in short-term mating success does not necessarily translate into an equally high variance in lifetime reproductive suc- cess (Clutton-Brock 1988). In age-structured populations of constant size, the ratio of $N_{\mathrm{eV}}$ to the number of breeding adults is expected to fall within the range 0.25-0.75 under most demographic conditions and is generally expected to be close to 0.5 (Nunney 1991, 1993, 1996; Nunney and Elam 1994; Waite and Parker 1996). To test this prediction, we performed additional simulations to estimate $N_{\mathrm{eV}} / N_{\text {adults' }}$ where $N_{\text {adults }}$ is the census number of breeding adults. Consistent with theoretical expectations, simulation-based estimates of $N_{\mathrm{eV}} / N_{\text {adults }}$ averaged $0.508(\mathrm{SD}=0.185,95 \% \mathrm{CI}=$ $0.274-0.854)$. The mean estimate of $N_{\mathrm{eV}} / N_{\text {adults }}$ for the Amboseli baboon population was considerably lower than similar estimates obtained for 10 other mammalian species (median $=0.66$, range $=0.42-1.27$; Table 6 ) .

One of the chief merits of the individual-based modeling approach is that it obviates the need for restrictive assumptions concerning age-independence of survival and fertility (Rockwell and Barrowclough 1995). However, the model does not consider two other factors that may be expected to further reduce $N_{\mathrm{eV}} / N$ : heritability of fitness (e.g., due to environmental covariance in reproductive success between parents and offspring) and population substructure (Nei and Murata 1966; Whitlock and Barton 1997; Nunney 1999).

Although $N_{\mathrm{eV}}$ is the parameter of interest for predicting the rate of loss of genetic variation, the dimensionless quantity $N_{\mathrm{eV}} / N$ provides a useful basis for comparing different populations (Frankham 1995) or for extrapolating locally obtained estimates of $N_{\mathrm{eV}}$ to broader portions of a species' range (provided that census numbers can be estimated). To translate the ratio $N_{\mathrm{eV}} / N$ into an estimate of $N_{\mathrm{eV}}$, it is important to substitute the appropriate value of $N$ in the denominator (Nunney and Elam 1994; Rockwell and Barrowclough 1995). In our individual-based simulation model, lifetime reproductive success was measured as the number of newborns produced by each member of a cohort of newborns. Simulations yielded nearly identical estimates of $N_{\mathrm{eV}} / N$ when lifetime reproductive success was measured over the course of an adult-to-adult life cycle (mean $N_{\mathrm{eV}} /$ $N=0.337, \mathrm{SD}=0.027,95 \% \mathrm{CI}=0.309-0.370)$. It does not necessarily matter which stage of the life cycle is used as a 
Table 3. Microsatellite allelic frequencies for Papio cynocephalus. For each locus, $n$ is the number of sampled chromosomes.

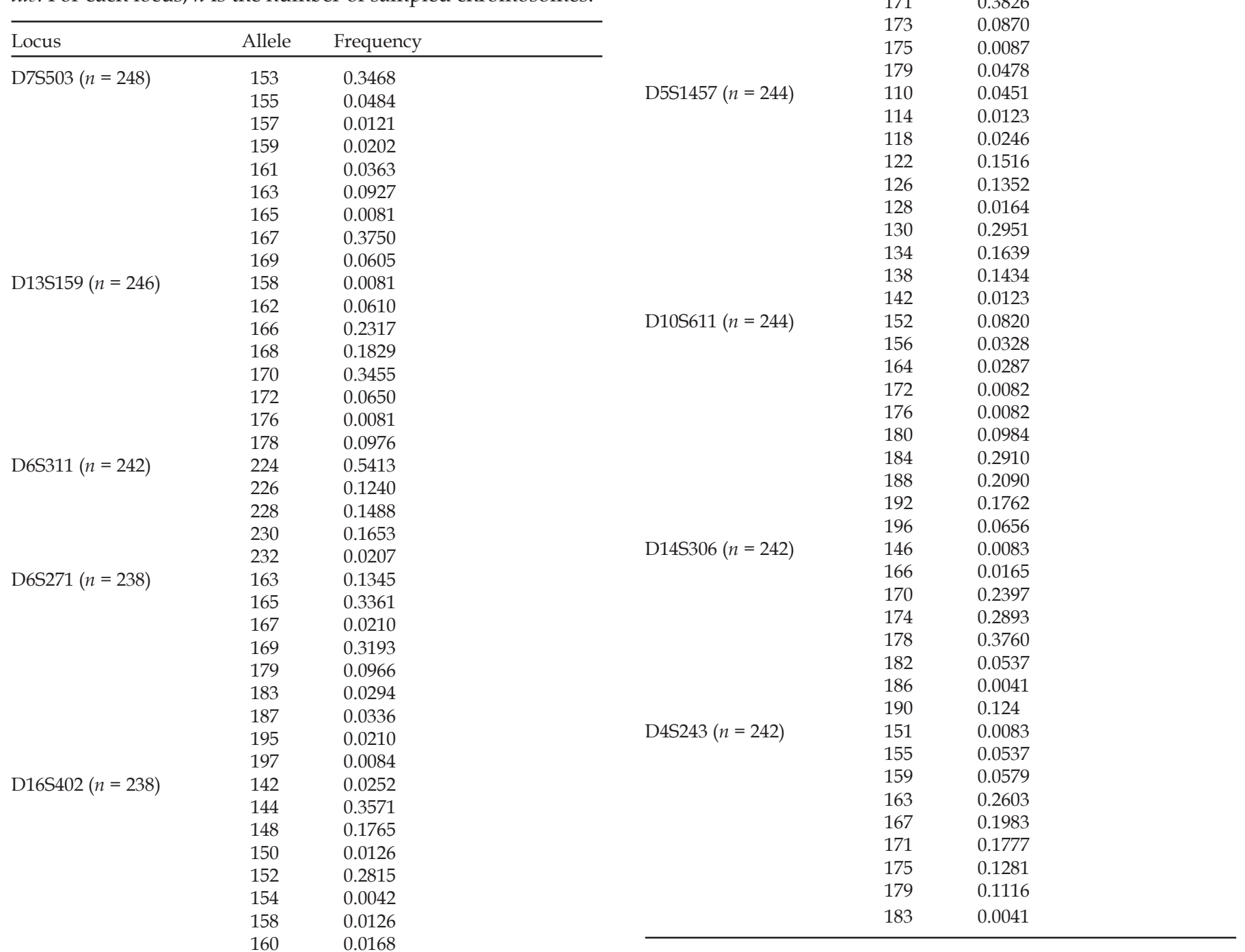

reference point for measuring lifetime reproductive success, as long as the denominator of the ratio $N_{\mathrm{eV}} / \mathrm{N}$ is chosen accordingly (Harris and Allendorf 1989; Nunney and Elam 1994; Rockwell and Barrowclough 1995). It should be noted that temporal fluctuations in population size can reduce long-term $N_{\mathrm{eV}} / \mathrm{N}$ well below the range of values predicted by models based on constant $N$ (Frankham 1995; Vucetich et al. 1997). Evidence for a past history of size fluctuations in the Amboseli baboon population is discussed below.

\section{Historical Demography and Long-Term $N_{e I}$}

Tests for evidence of a genetic bottleneck.

Detection of linkage disequilibria in the absence of any significant departures from Hardy-Weinberg genotypic proportions is not surprising given our knowledge of the recent demographic bottleneck. Randomly generated linkage disequilibrium is a more sensitive measure of the effects of sampling drift. A population will return to HardyWeinberg equilibrium after a single generation of random mating (or a relatively small number of generations in agestructured populations), whereas linkage disequilibrium is 
Table 4. Summary statistics derived from coalescent-based simulations of microsatellite evolution under the two-phase mutation model (TPM). Simulation results are presented separately for two different parameterizations of the TPM: proportions of multistep mutations $(q)$ were set at 0.05 and $0.10 . H_{\text {eq }}$ average expected heterozygosity at mutation-drift equilibrium; SD, standard deviation of single-locus $H_{\mathrm{eq}}$-values; $H_{\mathrm{e}}-H_{\mathrm{eq}}$, heterozygosity excess (if positive) or heterozygosity deficit (if negative); and $P$, probability that $H_{\mathrm{e}}>H_{\mathrm{eq}}$ under the null hypothesis of mutation-drift equilibrium.

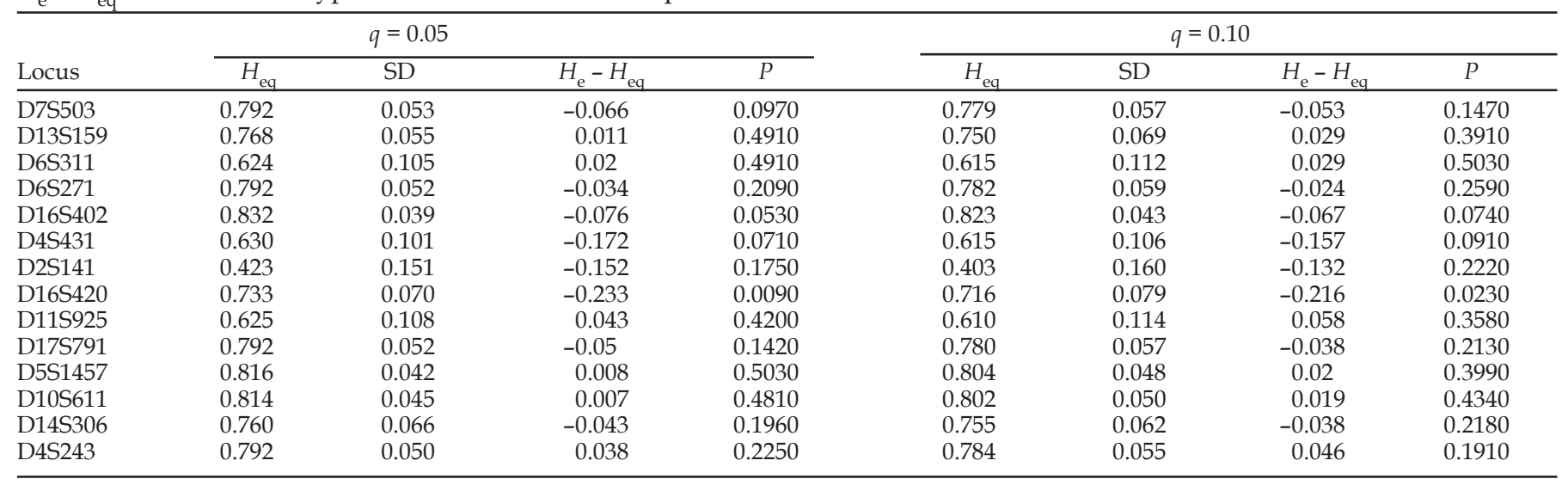

dissipated at a rate determined by the recombination fraction (0.5 for unlinked loci; Hartl and Clark 1997).

Tests based on allelic frequencies at microsatellite loci revealed no significant departures from mutation-drift equilibrium. Because mutation-drift equilibrium will be approximately reached if the effective size of a population remains stationary for $4 N_{\mathrm{eV}}$ to $10 \mathrm{~N}_{\mathrm{eV}}$ generations (Nei and Li 1976), the microsatellite data suggest that the Amboseli baboon population has remained relatively stable in recent history. This conclusion is obviously at odds with the welldocumented population crash in the late 1960s (Altmann et al. 1985; Samuels and Altmann 1991). The Amboseli population suffered a 90\% reduction, from about 2500 to 255 baboons within a 5-year period (1964-1969; Altmann et al. 1985). Why was this demographic bottleneck not reflected by any discernable reduction in $N_{\mathrm{ev}}$ ?

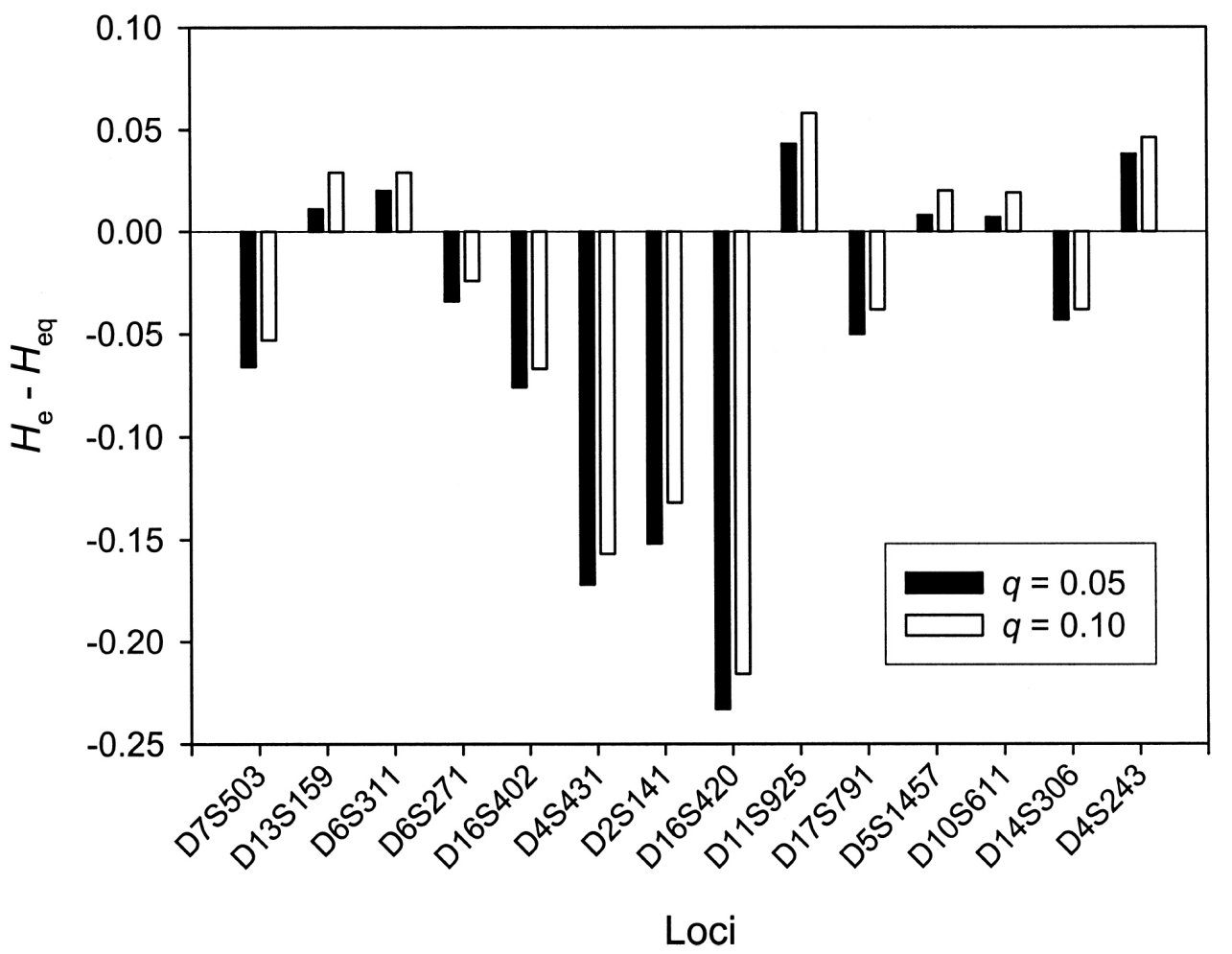

Figure 3. Patterns of heterozygosity excess $\left(H_{\mathrm{e}}-H_{\mathrm{eq}}>0\right)$ and deficiency $\left(H_{\mathrm{e}}-H_{\mathrm{eq}}<0\right)$ observed at each of 14 microsatellite loci in the Amboseli population of savannah baboons. The zero point on the y-axis denotes the expected value of $H_{\mathrm{e}}-H_{\mathrm{eq}}$ for neutral loci at mutation-drift equilibrium. Expected values were derived from coalescent-based simulations of microsatellite evolution under the two-phase mutation model with the proportion of multistep mutations $(q)$ set at 0.05 and 0.10 . 


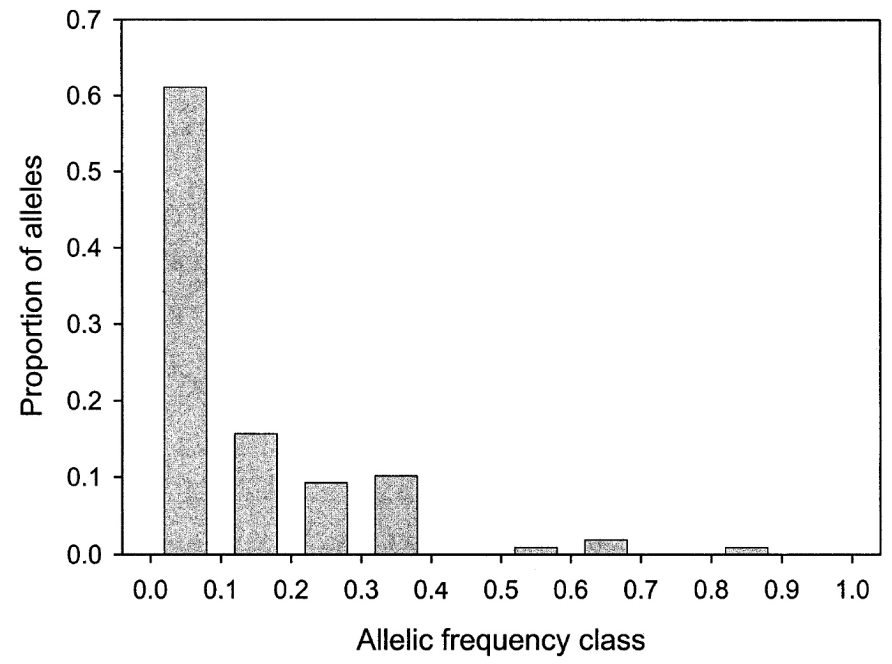

Figure 4. Distribution of allelic frequencies at 14 microsatellite loci from a sample of 136 savannah baboons. Allele counts conformed to the L-shaped frequency distribution expected for neutral loci at mutation-drift equilibrium (Luikart et al. 1998).

Populations suffering a reduction in census number (a demographic bottleneck) may not suffer a reduction of $N_{\mathrm{eV}}$ (a genetic bottleneck) if historical $N_{\mathrm{eV}}$ has always been low due to fluctuations in population size or extinction/recolonization dynamics (Vucetich et al. 1997; Whitlock and Barton 1997). However, this is not a likely explanation for the failure to detect evidence of a genetic bottleneck in the Amboseli baboon population. Maximum-likelihood estimates of $\theta$ indicate that the long-term historical $N_{\mathrm{eI}}$ of the Amboseli population is much larger than the contemporary census number. According to theory for the coalescent in fluctuating populations (Griffiths and Tavaré 1994b), longterm $N_{\mathrm{eI}}$ of a population subject to recurrent size changes is equal to the harmonic mean value through time. Although outside immigration may account for most of the effect (see below), the pronounced disparity between $N_{\mathrm{eI}}$ and contemporary census number suggests that the Amboseli population has been reduced far below historical levels.

It is also possible that the effects of drift could not be detected because insufficient time has elapsed since the onset of the population decline. Given that only three to four ba-
Table 5. Maximum-likelihood estimates of $\theta\left(=N_{\mathrm{eI}} \mu\right)$ and $q$ (proportion of multistep mutations) under the two-phase mutation model. Analysis was based on a total of 12 microsatellite loci with $H_{\mathrm{e}}$ in the range $0.50-0.90$ (see Table 2$) . l(\theta, q)$, joint likelihood of $\theta$ and $q$.

\begin{tabular}{lccc}
\hline Locus & $\theta$ & $q$ & $l(\theta, q)$ \\
\hline D7S503 & 4.221 & 0.010 & -40.222 \\
D13S159 & 8.929 & 0.010 & -39.841 \\
D6S311 & 2.377 & 0.010 & -21.844 \\
D6S271 & 5.733 & 0.182 & -50.938 \\
D16S402 & 5.075 & 0.476 & -50.073 \\
D16S420 & 3.729 & 0.108 & -31.218 \\
D11S925 & 2.475 & 0.010 & -15.288 \\
D17S791 & 8.783 & 0.059 & -37.687 \\
D5S1457 & 15.415 & 0.010 & -33.271 \\
D10S611 & 17.565 & 0.035 & -42.119 \\
D14S306 & 6.857 & 0.035 & -30.136 \\
D4S243 & 18.360 & 0.010 & -28.423 \\
\hline
\end{tabular}

boon generations had elapsed since the onset of the bottleneck, the analysis of Cornuet and Luikart (1996) indicates that the power to detect a 10 -fold reduction in population size may be quite low. It is therefore likely that the tests applied had insufficient power to detect the genetic signature of such a recent bottleneck. In any case, results of the microsatellite-based tests suggest that prior to the onset of the recent population decline, any past changes in population size were not of sufficient magnitude to perturb the population from mutation-drift equilibrium.

\section{Estimation of long-term $N_{e I}$.}

Levels of microsatellite polymorphism in humans and baboons are closely similar for loci segregating at least four alleles in each species, and allele sizes at homologous loci are strongly and positively correlated (Morin et al. 1998). Thus, in combination with maximum-likelihood estimates of $\theta$, use of pedigree-based estimates of mutation rates for human microsatellites with $H_{\mathrm{e}}$ in the same range as those used in our analysis (Brinkman et al. 1998) can be expected to yield reasonable estimates of long-term $N_{\mathrm{el}}$.

The coalescent-based estimate of historical $N_{\mathrm{eI}}$ was considerably larger than the contemporary census size of the Amboseli baboon population. This result would appear

Table 6. Demographic estimates of $N_{\mathrm{eV}} / N_{\text {adults }}$ for populations of 11 mammalian species. Estimates of $N_{\mathrm{eV}} / N_{\mathrm{adults}}$ for Crocidura russula, Homo sapiens, and Papio cynocephalus were obtained using equation (5). The remaining estimates were obtained using a simplified version of the Hill equation based on the assumption of age-independent survival and fertility (Nunney 1993; Nunney and Elam 1994). $T_{\mathrm{m}}$ and $T_{\mathrm{f}}$ are the mean generation times (in years) for males and females, respectively.

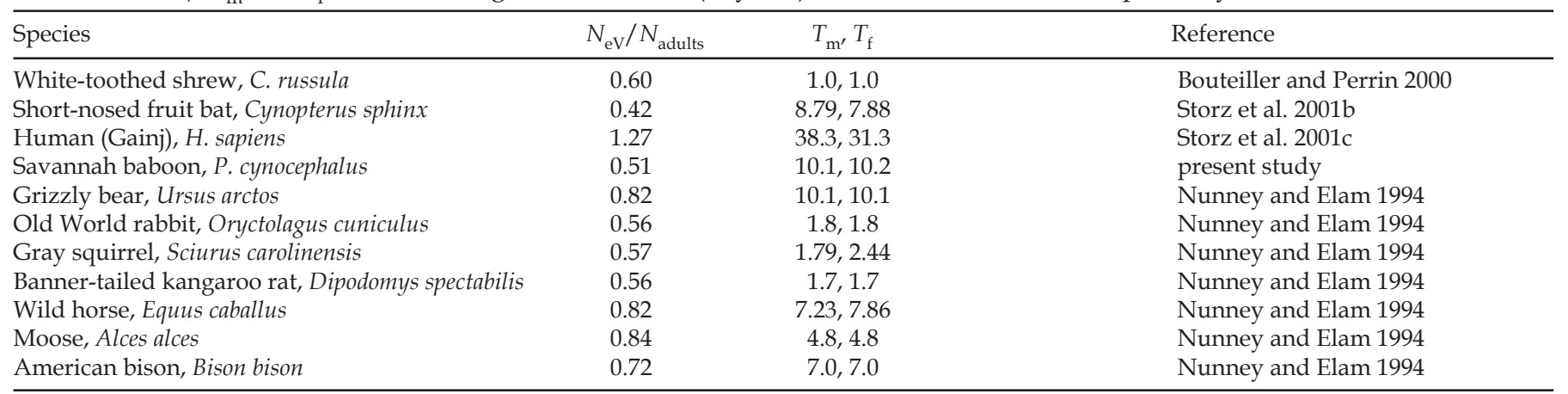


to be consistent with the suggestion that $N_{\mathrm{eI}}$ may often exceed the instantaneous census number of populations that are subdivided into a network of socially defined breeding groups (Sugg et al. 1996). In subdivided populations, $N_{\mathrm{eI}}$ may exceed the total census number because the amonggroup component of genetic variance is safeguarded from the sampling effects of Mendelian segregation in heterozygotes. At equilibrium in an island model of population structure, $N_{\mathrm{eI}}$ is increased above $N$ by the factor $1 /\left(1-F_{\mathrm{ST}}\right)$, where $F_{\mathrm{ST}}$ is the standardized among-deme variance in allelic frequencies (Wright 1943; Nei and Takahata 1993).

However, the effects of population subdivision and local inbreeding on $N_{\mathrm{eI}}$ are highly dependent on the nature of population regulation (Whitlock and Barton 1997; Nunney 1999). When productivity of breeding groups is equalized by local density regulation, $N_{\mathrm{el}}$ of the total population is reduced by inbreeding within groups $\left(F_{\mathrm{IS}}\right)$ and is increased by inbreeding among groups $\left(F_{\mathrm{ST}}\right.$; assuming a 1:1 sex ratio and nonoverlapping generations). Classic formulas describing the dynamics of sampling drift in an island model of population structure (Wright 1931, 1943) are based on the assumption of local density regulation. In other words, the models implicitly assume an ecological context in which variance in deme fitness is suppressed by soft selection (Wade 1985; Kelly 1994). This also holds true for extensions of the island model that incorporate complexities related to sex differences in patterns of mating and dispersal (Chesser et al. 1993; Wang 1997). In the absence of local density regulation (the interdemic model of Nunney 1999), variance in productivity of breeding groups produces an additional source of drift and reduces $N_{\mathrm{eI}}$ relative to expectations for a panmictic population with the same census number (Whitlock and Barton 1997; Nunney 1999; Wang and Caballero 1999). When interdemic drift acts in concert with local inbreeding, the effects of $F_{\mathrm{IS}}$ and $F_{\mathrm{ST}}$ are largely symmetrical: Increasing inbreeding either within or among breeding groups reduces $N_{\mathrm{eI}}$ of the total population. Thus, estimating the effect of population subdivision on $N_{\mathrm{eI}}$ requires information about the scope for drift due to among-group variance in progeny production (Whitlock and Barton 1997; Nunney 1999).

Rates of progeny production are indeed highly variable among matrilineal kin groups of savannah baboons (Altmann et al. 1985; Samuels and Altmann 1991). Thus, Nunney's (1999) theoretical results suggest that baboon social structure could be expected to reduce $N_{\mathrm{eI}}$ relative to panmictic expectations. However, until such theory is extended to age-structured populations, the expected effect of social structure on $N_{\mathrm{eI}}$ of primates and other long-lived mammals will remain uncertain. In any event, the genetic data revealed no evidence of population subdivision in our sample of Amboseli baboons. A significant degree of genetic subdivision among age cohorts or family groups would be reflected by a departure from Hardy-Weinberg genotypic proportions in the direction of a heterozygote deficit. No such Wahlund effect was detected in our population sample, as the overall inbreeding coefficent $(f=-0.016$, averaged across 16 loci) was in the direction of a heterozygote excess.

It is likely that the disparity between historical $N_{\text {eI }}$ and contemporary census size of the Amboseli baboon population is primarily attributable to current and/or historical immigration from outside the physiographic confines of the basin and to the time lag between the recent drop in census numbers and the rate of increase in the average probability of allelic identity-by-descent. Reduction in census numbers can occur very rapidly, whereas the increase in homozygosity due to sampling drift is generally a slower process. If the Amboseli baboon population were to become completely insularized, we would expect a further reduction in genetic diversity, and $N_{\mathrm{el}}$ should eventually converge on the census number. However, the Amboseli baboon population has not yet reached this point, and observed levels of genetic diversity may primarily reflect the population's prebottleneck history rather than its current demography.

\section{Acknowledgments}

We thank J. Altmann, F. S. Dobson, H. L. Gibbs, R. Lande, and one anonymous reviewer for constructive comments on the manuscript. We also thank G. Luikart and L. Nunney for helpful discussions and M. Bruford for sharing microsatellite data. This work was supported by a grant to SCA from the National Science Foundation (IBN0002342). The Office of the President of Kenya and the Kenya Wildlife Service kindly provided permission to work in Amboseli. We thank R. Leakey, J. Else, N. Kio, D. Western, and the staff of Amboseli National Park for cooperation and assistance. M. Isahakia, C. S. Bambara, J. Mwenda, O. Mushi, C. Mlay, the members of the pastoralist communities of Amboseli and Longido, and the Institute for Primate Research in Nairobi provided assistance and local sponsorship. JFS acknowledges support from an NRSA Postdoctoral Fellowship from the National Institutes of Health and a Fellowship in Computational Molecular Biology from the Alfred P. Sloan Foundation and U.S. Department of Energy.

\section{Literature Cited}

Alberts S. C., and J. Altmann. 1995. Balancing costs and opportunities: dispersal in male baboons. Am. Nat. 145:279-306.

Alberts S. C. 2002. Matrix models for primate life history analysis. In P. Kappeler and M. Pereira, eds. Primate life history and socioecology. Univ. of Chicago Press, Chicago, IL.

Allison P. D. 1995. Survival analysis using the SAS system: a practical guide. SAS Institute, Cary, NC.

Altmann J., G. Hausfater, and S. A. Altmann. 1985. Demography of Amboseli baboons, 1963-1983. Am. J. Primatol. 8:113-125.

Altmann J., S. C. Alberts, S. A. Haines, J. Dubach, P. Muruthi, T. Coote, E. Geffen, D. J. Cheesman, R. S. Mututua, S. N. Saiyalel, R. K. Wayne, R. C. Lacy, and M. W. Bruford. 1996. Behavior predicts genetic structure in a wild primate group. Proc. Natl. Acad. Sci. USA. 93:5797-5801.

Begon M., C. B. Krimbas, and M. Loukas. 1980. The genetics of Drosophila subobscura populations. XV. Effective size of a natural population estimated by three independent methods. Heredity. 45:351-359.

Bouteiller C., and N. Perrin. 2000. Individual reproductive success and effective population size in the greater white-toothed shrew Crocidura russula. Proc. R. Soc. Lond. B. 267:701-705.

Brinkman B., M. Klintschar, F. Neuhuber, J. Huhne, and B. Rolf. 1998. Mutation rate in human microsatellites: influence of the structure and length of the tandem repeat. Am. J. Hum. Genet. 62:1408-1415.

Bruford M. W., O. Hanotte, J. F. Y. Brookfield, and T. Burke. 1992. Multi- and single-locus DNA fingerprinting. Pp. 225-269 in A. R. Hoelzel, ed. Molecular analysis of populations: a practical approach. IRL Press, Oxford, U.K.

Caballero A. 1994. Developments in the prediction of effective population size. Heredity. 73:657-679. 
Cambefort Y., C. Mounie, and P. Colombies. 1976. Chromosome banding patterns of Papio papio. Ann. Genet. 19:5-9.

Caswell H. 2001. Matrix population models. 2d ed. Sinauer, Sunderland, MA.

Chakraborty R., and M. Nei. 1977. Bottleneck effects on average heterozygosity and genetic distance with the stepwise mutation model. Evolution. 31:347-356.

Chakraborty R., P. A. Fuerst, and M. Nei. 1980. Statistical studies on protein polymorphism in natural populations. III. Distribution of allele frequencies and the number of alleles per locus. Genetics. 94:1039-1063.

Chesser R. K., O. E. Rhodes Jr., D. W. Sugg, and A. Schnabel. 1993. Effective sizes for subdivided populations. Genetics. 135::1221-1232.

Clutton-Brock T. H. 1988. Reproductive success. Pp. 472-485 in T. H. Clutton-Brock, ed. Reproductive success. Univ. of Chicago Press, Chicago, IL.

Cockerham C. C. 1969. Variance of gene frequencies. Evolution. 23:72-84.

Cornuet J.-M., and G. Luikart. 1996. Description and power analysis of two tests for detecting recent population bottlenecks from allele frequency data. Genetics. 144:2001-2014.

Crandall K. A., D. Posada, and D. Vasco. 1999. Effective population size: missing measures and missing concepts. Anim. Cons. 2:317-319.

Crow J. F., and C. Denniston. 1988. Inbreeding and variance effective population numbers. Evolution. 42:482-495.

Crow J. F., and M. Kimura. 1970. Introduction to population genetics theory. Harper and Row, New York.

Di Rienzo A., C. Peterson, J. C. Garza, A. M. Valdes, M. Slatkin, and N. B. Freimer. 1994. Mutational processes of simple-sequence repeat loci in human populations. Proc. Natl. Acad. Sci. USA. 91:3166-3170.

Ellegren H. 2000. Microsatellite mutations in the germline: implications for evolutionary inference. Trends Genet. 16:551-558.

Emigh T. H., and E. Pollack. 1979. Fixation probabilities and effective population numbers in dipolid populations with overlapping generations. Theor. Popul. Biol. 15:86-107.

Estoup A., and J.-M. Cornuet. 1999. Microsatellite evolution: inferences from population data. Pp. 49-65 in D. B. Goldstein and C. Schlötterer, eds. Microsatellites: evolution and applications. Oxford Univ. Press, Oxford, U.K.

Ewens W. J. 1972. The sampling theory of selectively neutral alleles. Theor. Popul. Biol. 3:87-112.

Ewens W. J. 1982. On the concept of effective population size. Theor. Popul. Biol. 21:373-378.

Felsenstein J. 1971. Inbreeding and variance effective numbers in populations with overlapping generations. Genetics. 68:581-597.

Felsenstein J. 1992a. Estimating effective population size from samples of sequences: inefficiency of pairwise and segregating sites as compared to phylogenetic estimates. Genet. Res. Camb. 59::139-147.

Felsenstein J. 1992b. Estimating effective population size from samples of sequences: a bootstrap Monte Carlo integration method. Genet. Res. Camb. 60:209-220.

Frankham R. 1995. Effective population size/adult population size ratios in wildlife: a review. Genet. Res. Camb. 66:95-107.

Fu Y. 1994a. A phylogenetic estimator of effective population size or mutation rate. Genetics. 136:685-692.

Fu Y. 1994b. Estimating effective population size or mutation rate using the frequencies of mutations of various classes in a sample of DNA sequences. Genetics. 138:1375-1386.

Gerber A. S., and A. R. Templeton. 1996. Population sizes and withindeme movement of Trimerotropis saxatilis (Acrididae), a grasshopper with a fragmented distribution. Oecologia. 105::343-350.

Goudet J. 1995. FSTAT (Version 1.2): a computer program to calculate F-statistics. J. Hered. 86:485-486.

Griffiths R. C., and S. Tavaré. 1994a. Simulating probability distributions in the coalescent. Theor. Popul. Biol. 46:131-159.
Griffiths R. C. 1994b. Sampling theory for neutral alleles in a varying environment. Phil. Trans. R. Soc. Lond. B. 344:403-410.

Griffiths R. C. 1994c. Ancestral inference in population genetics. Stat. Sci. 9:307-319.

Guo S. W., and E. A. Thompson. 1992. Performing the exact test of Hardy-Weinberg proportion for multiple alleles. Biometrics. 48:361-372.

Harris R. B., and F. W. Allendorf. 1989. Genetically effective population size of large mammals: an assessment of estimators. Cons. Biol. 3:181-191.

Hartl D. L., and A. G. Clark. 1997. Principles of population genetics. 3rd ed. Sinauer, Sunderland, MA.

Higgins K., and M. Lynch. 2001. Metapopulation extinction caused by mutation accumulation. Proc. Natl. Acad. Sci. USA. 98::2928-2933.

Hill W. G. 1972. Effective size of populations with overlapping generations. Theor. Popul. Biol. 3:278-289.

Hill W. G. 1979. A note on effective population size with overlapping generations. Genetics. 92:317-322.

Hudson R. R. 1990. Gene genealogies and the coalescent process. Oxf. Surv. Evol. Biol. 7:1-44.

Husband B. C., and S. C. H. Barrett. 1992. Effective population size and genetic drift in tristylous Eichornia paniculata (Pontederiaceae). Evolution. 46:1875-1890.

Johnson D. L. 1977. Inbreeding in populations with overlapping generations. Genetics. 87:581-591.

Kalbfleisch J. D., and R. L. Prentice. 1980. The statistical analysis of failure time data. Wiley, New York.

Keller L. F., K. J. Jeffery, P. Arcese, M. A. Beaumont, W. M. Hochachka, J. N. M. Smith, and M. W. Bruford. 2001. Immigration and the ephemerality of a natural population bottleneck: evidence from molecular markers. Proc. R. Soc. Lond. B. 268::1387-1394.

Kelly J. K. 1994. The effect of scale dependent processes on kin selection: mating and density regulation. Theor. Popul. Biol. 46::32-57.

Kimura M., and J. F. Crow. 1963. The measurement of effective population number. Evolution. 17:279-288.

Kuhner M. K., J. Yamato, and J. Felsenstein. 1995. Estimating effective population size and mutation rate from sequence data using Metropolis-Hastings sampling. Genetics. 140:1421-1430.

Kuhner M. K. 1998. Maximum likelihood estimation of population growth rates based on the coalescent. Genetics. 149:429-434.

Lande R. 1995. Mutation and conservation. Cons. Biol. 9:782-791.

Lande R., and G. F. Barrowclough. 1987. Effective population size, genetic variation, and their use in population management. Pp. 87123 in M. E. Soulé, ed. Viable populations for conservation. Cambridge Univ. Press, Cambridge, U.K.

Leberg P. L. 1992. Effects of population bottlenecks on genetic diversity as measured by allozyme electrophoresis. Evolution. 46:477-494.

Lee E. T. 1992. Statistical methods for survival data analysis. Wiley, New York.

Luikart G., and J.-M. Cornuet. 1998. Empirical evaluation of a test for identifying recently bottlenecked populations from allele frequency data. Cons. Biol. 12:228-237.

Luikart G., F. W. Allendorf, J.-M. Cornuet, and W. B. Sherwin. 1998. Distortion of allele frequency distributions provides a test for recent population bottlenecks. J. Hered. 89:238-247.

Lynch M., J. Conery, and R. Bürger. 1995. Mutation accumulation and the extinction of small populations. Am. Nat. 146:489-518.

Maruyama T., and P. A. Fuerst. 1985. Population bottlenecks and nonequilibrium models in population genetics. II. Number of alleles in a small population that was formed by a recent bottleneck. Genetics. 111:675-689.

Mills S. L., and P. E. Smouse. 1994. Demographic consequences of inbreeding in remnant populations. Am. Nat. 144:412-431.

Morin P. A., P. Mahboubi, S. Wedel, and J. Rogers. 1998. Rapid screening and comparison of human microsatellite markers in ba- 
boons: allele size is conserved, but allele number is not. Genomics. 53:12-20.

Motro U., and G. Thomson. 1982. On heterozygosity and the effective size of populations subject to size changes. Evolution. 36:1059-1066.

Nei M. 1987. Molecular evolutionary genetics. Columbia Univ. Press, New York.

Nei M., and W.-H. Li. 1976. The transient distribution of allele frequencies under mutation pressure. Genet. Res. Camb. 28::205-214.

Nei M., and M. Murata. 1966. Effective population size when fertility is inherited. Genet. Res. Camb. 8:257-260.

Nei M., and N. Takahata. 1993. Effective population size, genetic diversity and coalescence time in subdivided populations. J. Mol. Evol. 37:240-244.

Nei M., T. Maruyama, and R. Chakraborty. 1975. The bottleneck effect and genetic variability in populations. Evolution. 29:1-10.

Nei M., R. Chakraborty, and P. A. Fuerst. 1976. Infinite allele model with varying mutation rate. Proc. Natl. Acad. Sci. USA. 73:4164-4168.

Nielsen R. 1997. A likelihood approach to population samples of microsatellite alleles. Genetics. 146:711-716.

Nunney L. 1991. The influence of age structure and fecundity on effective population size. Proc. R. Soc. Lond. B. 246:71-76.

Nunney L. 1993. The influence of mating system and overlapping generations on effective population size. Evolution. 47::1329-1341.

Nunney L. 1996. The influence of variation in female fecundity on effective population size. Biol. J. Linn. Soc. 59:411-425.

Nunney L. 1999. The effective size of a hierarchically structured population. Evolution. 53:1-10.

Nunney L., and K. A. Campbell. 1993. Assessing minimum viable population size: demography meets population genetics. Trends Ecol. Evol. 8:234-239.

Nunney L., and D. R. Elam. 1994. Estimating the effective size of conserved populations. Cons. Biol. 8:175-184.

Piry S., G. Luikart, and J.-M. Cornuet. 1999. BOTTLENECK: a computer program for detecting recent reductions in the effective population size using allele frequency data. J. Hered. 90::502-503.

Pollak E. 1980. Effective population numbers and mean times to extinction in dioecious populations with overlapping generations. Math. Biosci. 52:1-25.

Raymond M., and F. Rousset. 1995a. An exact test for population differentiation. Evolution. 49:1280-1283.

Raymond M. 1995b. GENEPOP (Version 1.2): population genetics software for exact test and ecumenicism. J. Hered. 86:248-249.

Rockwell R. F., and G. F. Barrowclough. 1995. Effective population size and lifetime reproductive success. Cons. Biol. 9::1225-1233.

Rogers J., M. C. Mahaney, S. M. Witte, S. Nair, D. Newman, S. Wedel, L. A. Rodriguez, K. S. Rice, S. H. Slifer, S. Perelygin, M. Slifer, P. Palladino-Negro, T. Newman, K. Chambers, G. Joslyn, P. Parry, and P. A. Morin. 2000. A genetic linkage map of the baboon (Papio hamadryas) genome based on human microsatellite polymorphisms. Genomics. 67:237-247.

Samuels A., and J. Altmann. 1991. Baboons of the Amboseli basin: demographic stability and change. Int. J. Primatol. 12:1-19.

Shriver M. D., L. Jin, R. Chakraborty, and E. Boerwinkle. 1993. VNTR allele frequency distributions under the stepwise mutation model: a computer simulation approach. Genetics. 134:983-993.

Storz J. F., H. R. Bhat, and T. H. Kunz. 2001a. Genetic consequences of polygyny and social structure in an Indian fruit bat, Cynopterus sphinx. I. Inbreeding, outbreeding, and population subdivision. Evolution. 55:1215-1223.
Storz J. F. 2001b. Genetic consequences of polygyny and social structure in an Indian fruit bat, Cynopterus sphinx. II. Variance in male mating success and effective population size. Evolution. 55:1224-1232.

Storz J. F., U. Ramakrishnan, and S. C. Alberts. 2001c. Determinants of effective population size for loci with different modes of inheritance. J. Hered. 92:497-502.

Sugg D. W., R. K. Chesser, F. S. Dobson, and J. L. Hoogland. 1996. Population genetics meets behavioral ecology. Trends Ecol. Evol. 11:338-342.

Summers K., and W. Amos. 1996. Behavioral, ecological, and molecular genetic analyses of reproductive strategies in the Amazonian dart-poison frog, Dendrobates ventrimaculatus Behav. Ecol. 8:260-267.

Tavaré S. 1984. Line-of-descent and genealogical processes, and their applications in population genetics models. Theor. Popul. Biol. 46:119-164.

Templeton A. R. 1980. The theory of speciation via the founder principle. Genetics. 94:1011-1038.

Templeton A. R., and B. Read. 1994. Inbreeding: one word, several meanings, much confusion. Pp. 91-105 in V. Loeschcke, J. Tomiuk, and S. K. Jain, eds. Conservation genetics. Birkhauser, Basel, Switzerland.

Valdes A. M., M. Slatkin, and N. B. Freimer. 1993. Allele frequencies at microsatellite loci: the stepwise mutation model revisited. Genetics. 133:737-749.

Vucetich J. A., T. A. Waite, and L. Nunney. 1997. Fluctuating population size and the ratio of effective to census population size. Evolution. 51:2017-2021.

Wade M. J. 1985. Hard selection, soft selection, kin selection, and group selection. Am. Nat. 125:61-73.

Waite T. A., and P. G. Parker. 1996. Dimensionless life histories and effective population size. Cons. Biol. 10:1456-1462.

Wang J. 1997. Effective size and F-statistics of subdivided populations. II. Dioecious species. Genetics. 146:1465-1474.

Wang J., and A. Caballero. 1999. Developments in predicting the effective size of subdivided populations. Heredity. 82:212-226.

Watterson G. A. 1984. Allele frequencies after a bottleneck. Theor. Popul. Biol. 26:387-407.

Watterson G. A. 1986. The homozygosity test after a change in population size. Genetics. 112:899-907.

Wehrhahn C. 1975. The evolution of selectively similar electrophoretically detectable alleles in finite natural populations. Genetics. 80:375-394.

Western D. 1983. A wildlife guide and natural history of Amboseli. General Printers, Nairobi, Kenya.

Western D., and C. van Praet. 1973. Cyclical changes in the habitat and climate of an East African ecosystem. Nature. 241:104-106.

Whitlock M. C. 2000. Fixation of new alleles and the extinction of small populations: drift load, beneficial alleles, and sexual selection. Evolution. 54:1855-1861.

Whitlock M. C., and N. H. Barton. 1997. The effective size of a subdivided population. Genetics. 146:427-441.

Wood J. W. 1987. The genetic demography of the Gainj of Papua New Guinea. 2. Determinants of effective population size. Am. Nat. 129:165-187.

Wright S. 1931. Evolution in Mendelian populations. Genetics. $16:: 97-159$.

Wright S. 1938. Size of population and breeding structure in relation to evolution. Science. 87:430-431.

Wright S. 1943. Isolation by distance. Genetics. 28:114-138. 